\title{
Enhanced Superframe Structure of the IEEE802.15.4 Standard for Real-time Data Transmission in Star Network
}

\author{
Khalid EL GHOLAMI \\ STIC, Chouaib Doukkali University \\ ElJadida, MOROCCO \\ LIMOS UMR 6158 CNRS, Blaise \\ Pascal University \\ Clermont-Ferrand, France
}

\author{
Kun-Mean HOU \\ LIMOS UMR 6158 CNRS, Blaise \\ Pascal University \\ Clermont-Ferrand, France
}

\author{
Najib ELKAMOUN \\ STIC, Chouaib Doukkali University \\ ElJadida, MOROCCO
}

\begin{abstract}
The IEEE 802.15.4 standard is one of the main communication protocols proposed for wireless sensor networks, IoT 'Internet of Things' and WoT 'Web of Things'. This protocol provides a flexible MAC layer designed to meet a variety of applications. Since WSN is application-specific, it's very difficult to provide a generic solution for all types of applications and topologies. In this work we focus on delay sensitive applications in star networks. This topology is used in wireless sensor networks for monitoring and control applications. The IEEE 802.15.4 standard provides some quality of service features for real-time data transmission. We identified some limitations of this standard and we proposed an improvement to provide a lower end-to-end delay with respect to energy consumption constraint by optimizing MAC layer. The experimentations are done using the NS-2 simulator. The results show the improvements expected by our approach among the IEEE 802.15.4 MAC standard.
\end{abstract}

\section{General Terms}

Quality of service, wireless sensor network, IEEE 802.15.4, Medium Access Control

\section{Keywords}

IEEE 802.15.4, WSN, Superframe, star topology, delay, Duty cycle, GTS

\section{INTRODUCTION}

Wireless sensor network has encountered a tremendous improvements thanks to the advances in MEMS (MicroElectro-Mechanical System), integrated circuit and wireless communication technologies. This technology can serve a vast range of applications. In our work we focus on applications that have short delay constraint in the star topology such as surveillance or monitoring. According to [1],[2] these applications will be retained for a long time. The star topology is an important topology specified by the IEEE 802.15.4 [3] standard. In this topology the coordinator manages the whole network and all data transmitted to any destination have to pass through the coordinator. Basically, for normal data, the network uses the CSMA/CA as a medium access control algorithm. But for the real-time data, the standard provides the ability to reserve some time slots to reduce medium access delay and to guarantee a constant bandwidth since the realtime data is sent without contentions.

Many research works have proposed solutions for different problems related to the GTS 'Guaranteed Time Slot' transmission by enhancing different mechanisms of the standard: GTS allocation management, superframe structure and GTS request management. Some of these works are discussed in related work section.

In this paper we propose an enhancement for the IEEE 802.15.4 standard superframe structure. We located some problems related to the standard GTS usage due to the current superframe structure, especially when the difference between BO 'Beacon Order' and SO 'Superframe Order' is high (in other words, low duty Cycle). In this kind of applications, the node may remain inactive for a long time which may increase the latency. In the reserved slots, communication is only allowed between the end device and its coordinator. When data is to be sent to another node in the same star network, it's sent first to the coordinator, which stores it and then sends it in the next superframe. The storage time relies on the sleep time which in typical WSN applications is supposed to be very high (very low duty cycle). Our proposal tends to reduce this additional delay by providing the possibility to send and receive real-time data in the same superframe, which makes our GTS usage less aware of the sleep time. Accordingly, the end devices may send critical data in short time and go to sleep to save power.

The rest of the paper is organized as follows. Section 2 reviews the IEEE802.15.4 MAC standard. In section 3, we present an overview of the related works. Section 4 identifies some problems in the current standard and presents the proposed solutions. In section 5 the solution for these problems is evaluated and we finish this paper by a conclusion and some perspectives for future works.

\section{THE IEEE802.15.4 MAC STANDARD}

\subsection{Overview of the IEEE 802.15.4 MAC protocol}

IEEE 802.15.4 standard specifies the physical layer (PHY) and the medium access control sub-layer (MAC) for low rate wireless personal area network. In the following we will describe the IEEE802.15.4 MAC sub-layer since our proposal is an enhancement of it.

The MAC sub-layer may operate in two alternative modes: beaconless mode and beacon enabled mode. In the beaconless mode, the nodes use only the unslotted CSMA/CA protocol to manage channel access and avoid collisions. This modified version of the standard CSMA/CA algorithm doesn't use the RTS/CTS mechanism to resolve the hidden terminal problem since the frame size is supposed to be very small. No 
synchronization is needed and no Quality of Service 'QoS' mechanism is expected in this mode which makes it suitable for application without QoS constraint. In this beacon-enabled mode the network uses a superframe to control the channel access. The superframe structure described in Figure 1 may be divided into two portions; the active portion and the inactive portion (sleep period). The active portion of the superframe may be divided into two periods: 1) contention access period (CAP), where network nodes use the slotted version of the CSMA/CA algorithm to contend for channel access. 2) Collision free period (CFP) where the channel is reserved and can be used exclusively by the reserving node using a slot labeled Guaranteed Time Slot 'GTS'. The CFP period is optional and used by low-latency applications or applications requiring specific data bandwidth. The sleep portion is also optional and it is used by networks where nodes don't need to be awake all the time.

In beacon enabled mode, the entire network is supervised by a central node called PAN 'Personal Area Network' Coordinator by advertising periodically a packet called beacon at the beginning of the superframe. The beacons are used to synchronize the attached devices, to identify the PAN, and to describe the structure of the superframe. It contains may also provide additional information about the pending addresses and the GTS configuration if needed. The superframe timing relay on three main parameters: beacon order (BO), the superframe order (SO) and the Final CAP Slot. These parameters are included in the beacon frame superframe specification field which allows the network nodes to define the superframe structure (the active period, The Contention Access Period 'CAP' length, the sleep time duration and the slot duration. Equations I, II and III are used for these calculations:

$\mathrm{BI}=\mathrm{aBaseSuperframeDuration} \times 2 \mathrm{BO}$ (symbols)

$\mathrm{SD}=$ aBaseSuperframeDuration $\mathrm{x} 2 \mathrm{SO}$ (symbols)

$\mathrm{sd}=\mathrm{aBaseSlotDuration} \times 2 \mathrm{SO}$ (symbols)

$$
\text { for } 0 \leq \mathrm{SO} \leq \mathrm{BO} \leq 14
$$

Where aBaseSuperframeDuration and aBaseSlotDuration are two constants predefined by the standard as 960 and 60 symbols respectively and denote the minimum length of the superframe and the slot respectively. Each symbol corresponds to 4 bits. BI (beacon interval) is the length of the whole superframe (including active period and inactive period). It is bounded by two beacon transmissions. The SD (superframe duration) represents the active period duration. And the 'sd' (slot duration) is the sixteenth of the active period (since SD is divided into 16 equally sized slots.)

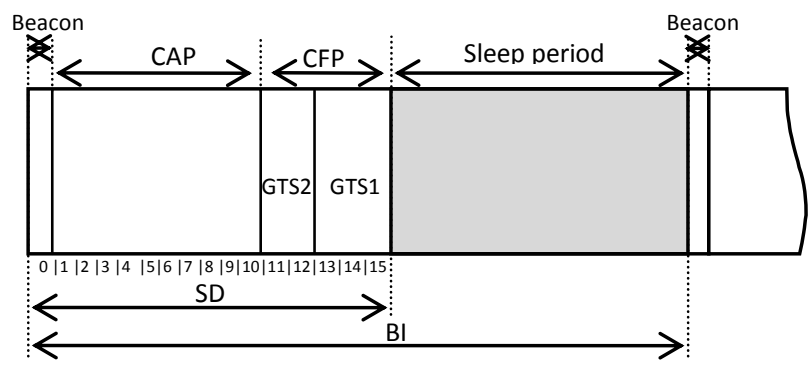

Figure 1: IEEE802.15.4 superframe structure

\subsection{GTS mechanism}

In the beacon-enabled mode, the PAN coordinator allows the other network nodes to reserve a dedicated time slots to satisfy the bandwidth and latency requirements via a TDMAlike 'Time Division Multi Access' medium access method. These slots are labeled as Guaranteed Time Slots 'GTS'. Each node can allocate up to two GTSs (one for receive and one for transmit), and one GTS may have more than one slot. The number of GTSs cannot exceed seven. These contiguous time slots form a Contention Free Period (CFP) which is placed at the end of the active period of the superframe. To use the GTS, the node has to send a GTS request to the PAN coordinator in the CAP (Contention Access Period), and when accepted the coordinator will advertise in its beacon all the information related to the GTS allocation. The node has to keep tracking the beacon for any possible changes (deallocation or reallocation). If the node does not receive the beacon, it is not allowed to use its GTS and has to wait for the next beacon. The transmission during the GTS is indirect (i.e. data has to go through the coordinator, and then the coordinator advertises the pending address in the beacon so that the destination can poll it by sending a data request MAC command). The GTS deallocation can be initiated by the allocating node or by the coordinator.

\subsection{Duty cycle:}

Energy is a critical resource in wireless sensor networks. Many MAC layer for sensor network were designed to reduce the power consumption, for example, S-MAC [4], T-MAC [5], U-MAC [6] and OD-MAC [7] are duty cycle based MAC protocols that can specify sleep and wake up times for network nodes within the frame. The IEEE 802.15.4 provide also a mechanism for power saving. This feature is possible only in beacon-enables mode when the $\mathrm{BO}$ is different than the SO $(\mathrm{SO}<\mathrm{BO})$. This sleep-awake scheme is suitable for wireless sensor networks since the nodes do not need to stay awake all the time, they may operate for a short time to send or receive collected data. This mechanism allows the devices to save power during sleep time. The PAN coordinator is the only device that may have to stay active all the time if it manages many clusters (cluster tree topology), in that case it should be mainly powered.

According to the standard, typical applications for IEEE 802.15.4 devices are anticipated to run with a very low duty cycles (under $1 \%$ ). If the duty cycle is not set correctly (e.g. too short), it may increase the transmission latency. Since during sleep time, data may have to wait until the active portion of the next superframe to start the transmission. This time can be computed as the ratio between the superframe duration and the beacon interval that can be related to $\mathrm{BO}$ and SO via the following equation:

$\mathrm{DC}=\mathrm{SD} / \mathrm{BI}=2 \mathrm{SO}-\mathrm{BO}$ :

\section{RELATED WORKS}

The GTS mechanism of the IEEE 802.15.4 standard provides a reliable communication, but it is hard to satisfy the needs of various applications. For this reason, many improvements were proposed $[8,9,10,11,12,13,14,15,16,17,18]$. [8] extended the superframe structure to increase the number of GTS. The aim is to reduce the waste of channel bandwidth and to enhance the QoS support for multiple devices, authors in [9] divided the GTS length to slots smaller than a standard superframe slot to minimize the waste of the channel bandwidth. In [10] an implicit GTS allocation mechanism (iGAME) is proposed by providing the possibility to share the GTS by several nodes in round-robin way. [11] proposes a D-GTS via a new dynamic GTS allocation algorithm for the periodic data transmission as well as the efficient use of the GTS slots. These dynamic GTSs are allocated at regular 
intervals in the contention access period. The superframe backoff period unit is used to determine the length of these GTS in spite of using the superframe slot unit as defined in the standard. With smaller slots, GTS utilization is expected to be more efficient than that in the standard scheme. [12] Proposes a method to resolve the insufficient GTS slot problem by allocating the GTS with higher priority first. The GTS requests are classified according to their priorities which allows GTSs to be allocated first for nodes those have realtime data by giving them higher priorities. It overcomes the under utilization of GTS bandwidth and the number of the concurrently allocable GTSs. [13] proposed a fully deterministic MAC protocol that supports a predefined time slots used for real-time association. This new scheme tends to avoid unsuccessful GTS request and to avoid also the collision during the GTS between nodes in different star in the same transmission range. Other works [14][16][15][17][18] proposed some improvement to the CSMA/CA mechanism to provide QoS support for real-time data.

Most of these algorithms and improvements can be easily adapted to our new superframe scheme to improve and optimize the GTS usage.

\section{OUR PROPOSAL}

\subsection{Defining the problem and the context}

In our work we focus on applications where the communication occurs between nodes in the same star topology. According to the IEEE802.15.4 standard all the communications has to go through the coordinator, and the data has to be stored in the coordinator until being advertised in the beacon of the next superframe, the destination end device sends a data request command to poll the pending data.

One of the weak points of the GTS usage in this kind of scenarios is that the real-time data is sent at the end of the superframe to the coordinator, thus the destination end device have to wait until the next beacon transmission (next superframe) to request this data. The reception is done using slotted CSMA/CA which may not guarantee the access to the medium. Also, since in wireless sensor networks it's much recommended to set a low duty cycle (high sleep time), the data may be stored in the coordinator for at least a time equal to the sleep period duration (equation 5) which may be very high. In Figure 2, the graph shows the evolution of the sleep time over different $\mathrm{BO}$ values ( $\mathrm{SO}$ is fixed to 4 in this example). This sleep time is translated to an additional delay that can be very high (e.g. 251 seconds for $\mathrm{SO}=4$ and $\mathrm{SO}=14$ ). Table 1 gives more detailed information for different values of BO and their corresponding sleep time and duty cycle.

$$
\text { Sleep delay }=\text { BI }- \text { SD }
$$

Table 1: sleep time and duty cycle for different $\mathrm{BO}$ values ( $\mathrm{SO}=4$, PHY 2.4G (250kbps))

\begin{tabular}{|c|c|c|c|c|c|}
\hline SO & $\mathbf{5}$ & $\mathbf{6}$ & $\mathbf{7}$ & $\mathbf{8}$ & $\mathbf{9}$ \\
\hline Sleep time (s) & 0,37 & 0,73 & 1,72 & 3,68 & 7,61 \\
\hline Duty Cycle & $50 \%$ & $25 \%$ & $12,5 \%$ & $6,25 \%$ & $3,13 \%$ \\
\hline \multirow{6}{*}{} & $\mathbf{1 0}$ & $\mathbf{1 1}$ & $\mathbf{1 2}$ & $\mathbf{1 3}$ & $\mathbf{1 4}$ \\
\cline { 2 - 6 } & 15,48 & 31,21 & 62,66 & 125,7 & 251,53 \\
\cline { 2 - 6 } & $1,56 \%$ & $0,78 \%$ & $0,39 \%$ & $0,20 \%$ & $0,1 \%$ \\
\hline
\end{tabular}

The other limitations are related to the current superframe structure itself. The first problem is that even if the node has reserved a GTS, it can contend for channel access in the CAP period which decreases the performance of the other nodes.
The second limitation is scheduling the CFP at the end of the active portion of the superframe. This scheme gives the normal data a faster channel access than the real-time data, since the real-time data may wait until the end of the CAP to get deterministic channel access.

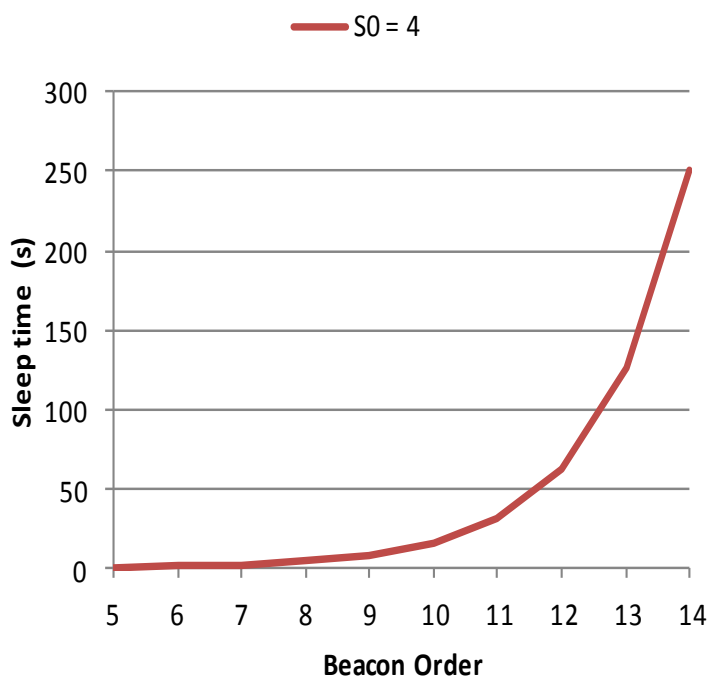

Figure 2: Sleep time for different $\mathrm{BO}$ values $(\mathrm{SO}=4$, 250kbps)

\subsection{Proposal}

In this section we describe our approach to resolve all these problems. We proposed a new superframe structure that allow a faster access to the channel and that avoid a high additional delay caused by the sleep time.

For the best of our knowledge, no method was proposed to solve the problems described above. This proposal tends to minimize the end-to-end delay when considering a very low duty cycle by sending and receiving the real-time data in the same superframe.

The new superframe has the same periods defined by the IEEE 802.15.4 standard (i.e. contention access period, contention free period and sleep time). The beacon is also sent at the beginning of the superframe and contains the information about the new superframe; the only field changed is the "Final CAP Slot" which was renamed to "Start CAP Slot". The new superframe described in Figure 3 is based on the idea to place the CFP after the beacon transmission. The CAP is placed between the end of CFP (or from the "Start CAP Slot") and the end of the active portion. After the CAP, nodes can go to sleep to save power if the coordinator allows it. This new scheme is very important and gives three improvements. 1) Nodes with real-time data can access the channel faster than those having normal data, since they don't need to wait for the end of the CAP to send their data. 2) The real-time nodes don't need to contend for the channel access in the CAP, since they send all their data in the CFP period which is placed at the beginning of the superframe. This new scheme may improve the performance of the other nodes and decrease the bandwidth and energy wastage due to unnecessary contentions. 3) The third improvement is very important since it is related to the energy-delay tradeoff. In this proposal we give the possibility to the real-time data to be sent and received in the same superframe. This allows avoiding the additional delay caused by storing data in the 
coordinator during sleep time. For this reason we adopted the same enhanced superframe structure since the CFP is placed in the beginning of the active portion. After the CFP is finished, the coordinator send an advertisement to the network nodes concerning the new packets sent in the previous period. For this purpose we created a new packet labeled Pending Real-Time Packets Update 'PRTPA'. For this section, the coordinator uses the simple algorithm 1. Thereby the destination nodes will know about the real-time pending data and will send a data request command to the coordinator to poll this data in the CAP of same superframe. The packet exchange timing is described in Figure 4

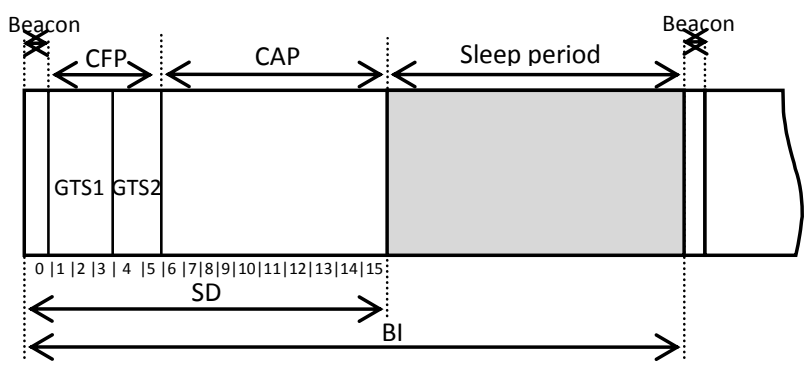

Figure 3 : Enhanced superframe structure

For the sending end device, the operation is quite the same as the standard, since the end device with critical data will send its packets during its GTS as described in the standard, the only difference is that the CFP is placed after the beacon transmission.
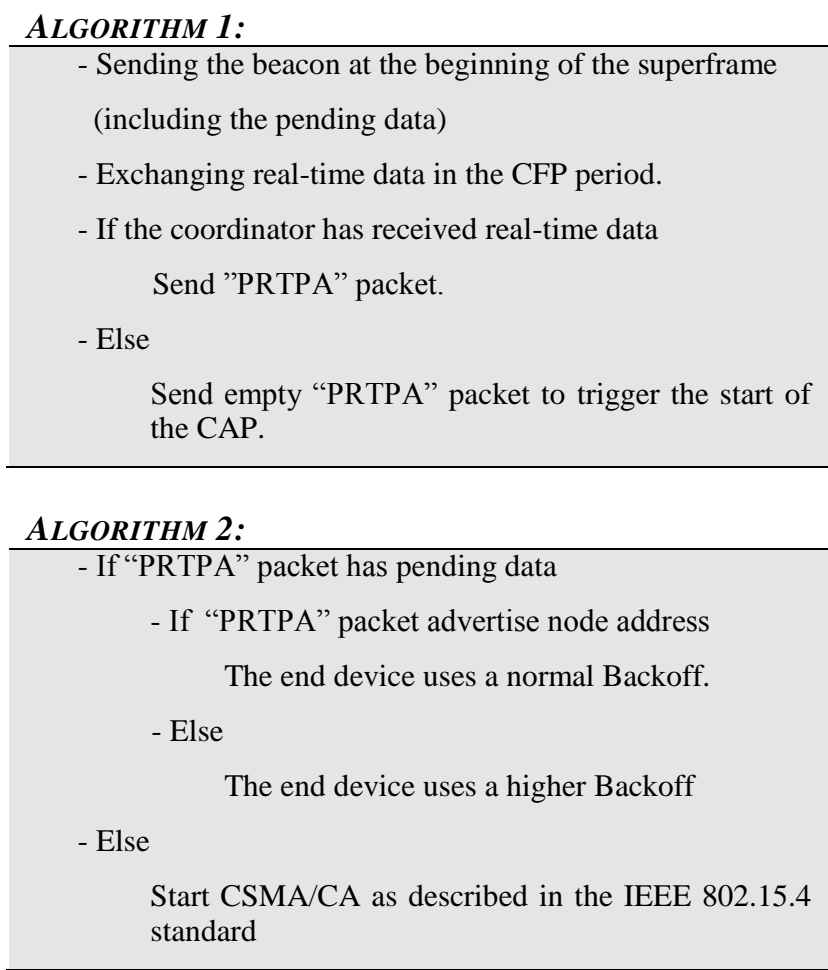

The end devices will wait for the PRTPU 'Pending Real-Time Packets Update' packet to start the CAP. When received, the end devices use the algorithm 2. All nodes that have pending data including real-time data are going to be polled in this period. This new scheme gives the opportunity to the real- time data to be transmitted and received in the same superframe. However, since only CSMA/CA is used in the CAP period, there is no guarantee that the packet will be transmitted in the same superframe. In this regard we proposed to use differentiate between different pending data type using different backoff exponent ' $\mathrm{BE}$ ' $\left(\mathrm{BE}_{\text {Real-time }}\right.$ is less than $\left.\mathrm{BE}_{\text {Others }}\right)$. This is easily managed since all nodes will receive the Beacon and RTPU and will know the pending data type (normal packets pending list is sent in the beacon and the critical data packets are sent in the RTPU packet); Each node that has pending real-time data will use a normal backoff to send a data request MAC command. Otherwise they will use a longer backoff exponent. If there is no real-time data (i.e. empty "PRTPA" packet sent), all nodes will use the standard backoff.

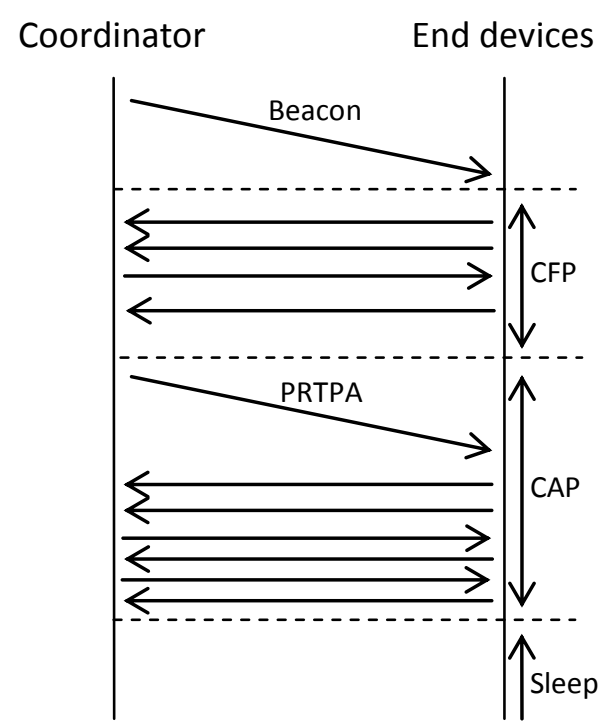

Figure 4 : Message sequence chart describing the packet exchange timing

\section{Performance evaluation}

The performance evaluation is based on the WPAN 'Wireless Personal Area Network' model [18] included in NS-2 simulator [19] (version 2.34). This model is good and was used in several papers to evaluate either the performance the IEEE 802.15.5 standard or the performance of their own proposed improvements. One of the limitations of this model is that it doesn't support the GTS mechanism. Accordingly, part of our work was to complete the WPAN model by the implementation of the GTS mechanism. Then our approach was implemented and compared to the IEEE 802.15.4 MAC standard.

The simulations make the following assumptions. The IEEE802.15.4 MAC layer operates in beacon enabled mode and all packets require MAC layer acknowledgement. The physical layer provides $250 \mathrm{kbps}$ and operates on $2.4 \mathrm{GHz}$ band. The network topology used in the simulation is a star topology. The traffic load is varied by changing the number of simulation nodes. The scenarios contain the PAN coordinator which is placed in the center of the star network to reach all the network nodes and a variable number of nodes randomly distributed in a $15 \mathrm{~m}$ radius circle. No routing protocol is used since we only use star topologies and we aim to evaluate our approach without any influence of the upper layers. In our simulation we disabled ARP (Address Resolution Protocol) 
since it's not needed in ZigBee networks. The application layer uses UDP packets with data rate of one packet each BI (Beacon Interval) since in real word, the BO may be chosen depending on the sensing frequency. The Tables 2 summarizes different simulation parameters for different scenarios. Figure 5 shows an example of 51 nodes topology.

We run multiple simulations for a $\mathrm{SO}=4$ and $\mathrm{SO}=5$ and different $\mathrm{BO}$ values $(4,5,6,7,8,9$ and 10). Our approach is compared to the GTS mechanism of the IEEE 802.15.4 MAC standard.

The network performance metrics used in this study are latency and BO (duty cycle). The latency is purely caused by medium access delay which is represented by the time when the packet is stored in MAC layer queue before transmission. The propagation delay is neglected and there is no latency caused by the upper layer (mainly network layer) since it's not used.

Tableau 2 : summary of the simulations parameters

\begin{tabular}{|c|c|}
\hline Parameters & Value \\
\hline Number of end devices & $10,20,30,40,50,60,70$, \\
& Star \\
\hline Topology & 50 bytes \\
\hline Packet size & 2000 seconds \\
\hline Simulation time & $15 \mathrm{~m}$ \\
\hline Transmission range & One packet every BI \\
\hline Transmission rate & Deactivated \\
\hline Routing protocol & More than 112 \\
\hline ARP & \\
\hline Number of scenarios &
\end{tabular}

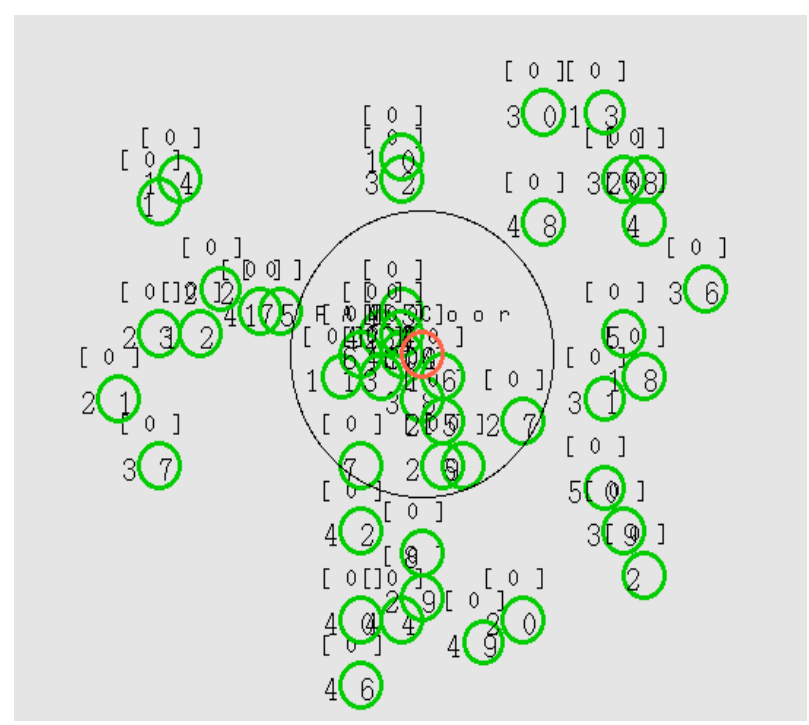

Figure 5: Performance evaluation topology

These simulations results highlight many important points. First we notice that the end-to-end delay measured in all scenarios follow the same appearance showed in Figure 2 with higher delay since the reception is done using CSMA/CA algorithm. This illustrates exactly the problem described in section 4 , which relate the end-to-end delay to the sleep time duration. Second, different result shows that our approach provides a better delay performance than the original GTS mechanism of the IEEE 802.15.4 standard.
In Figure 6, the $\mathrm{x}$ axis shows the different values of the beacon order while the measured the end-to-end delay appears on the y axis. SO is being fixed to 5 and the network contains 20 end devices and one PAN coordinator. It may be seen clearly that our approach provides a lower end to end delay than the standard GTS mechanism for all BO values. This is exactly what was excepted since our approach provides the possibility to send and receive the real-time packets in the same superframe.

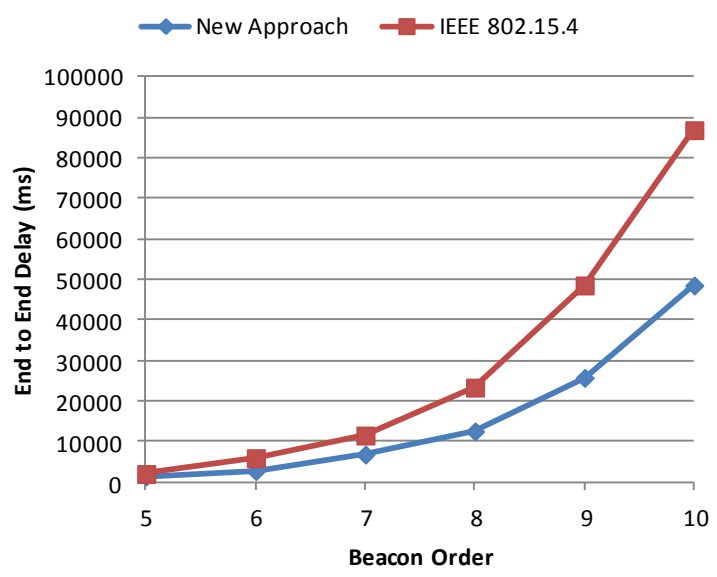

Figure 6: End-to-End delay vs. beacon order (21 nodes, SO=5)

Our proposal gives also better performance as shown in Figure 7 with a different number of nodes (30 end devices and one PAN coordinator) and by changing the $\mathrm{SO}$ which in this case is equal to 4 . The delay was increased because the reception is done using CSMA/CA which is heavily influenced by the node density. Our algorithm gives in this scenario also a lower end-to-end delay.

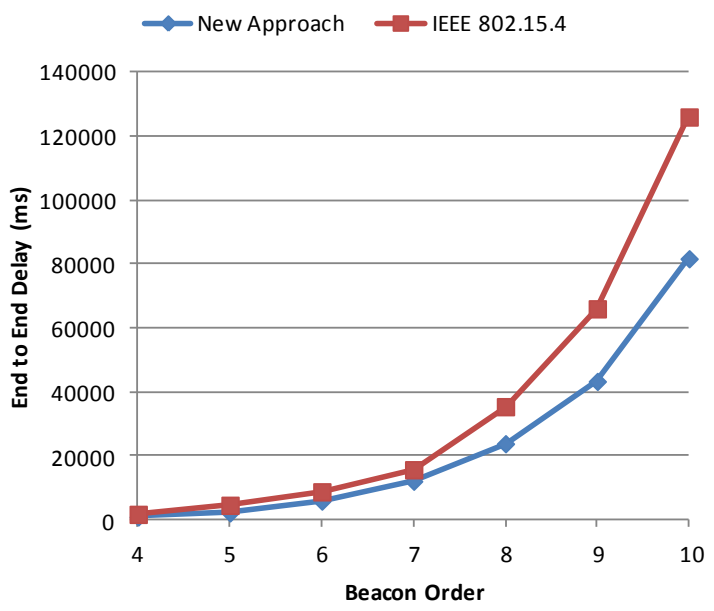

Figure 7: End-to-End delay vs. beacon order (31 nodes, $\mathrm{SO}=4)$

In Figure 8, the graph shows the influence of the duty cycle on the End-to-End for a higher number of nodes (50 end devices and one coordinator). We can easily see the influence of the duty cycle on the delay, our approach provide also a better performance for all duty cycles compared to the current GTS mechanism if the IEEE 802.15.4 standard. 


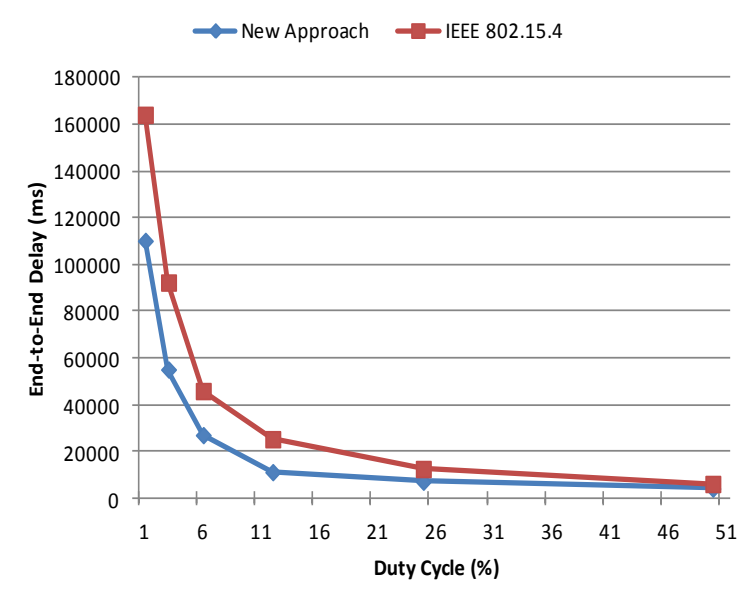

Figure 8: End-to-End delay vs. duty cycle (51 nodes)

In Figure 9 we compared the end to end delay for different traffic loads (i.e. different numbers of nodes). This test is done for a duty cycle of $1.56 \%(\mathrm{BO}=10, \mathrm{SO}=4)$. The results show that our algorithm provides better performance than the IEEE 802.15.4 MAC layer among all these simulation scenarios and nodes densities

In figure 10, we summaries all the experiments we have done using the new approach by fixing SO to 4 and changing $\mathrm{BO}$ from 4 to 10 and by varying the number of network nodes from 10 to 80 nodes. The results showed that the end-to-end delay may be influenced by the length of the sleep time and traffic load. This impact is logic since the reception is done using the CSMA/CA algorithm with a differentiation between real time data and normal data. However, if compared to the current GTS mechanism of the IEEE 802.15.4 standard, the delay is considerably reduced by our approach for different BO and nodes numbers as we proved in the previous results. These experimentations validate the improvements expected by our new superframe structure that allow sending and receiving real time data within the same superframe.

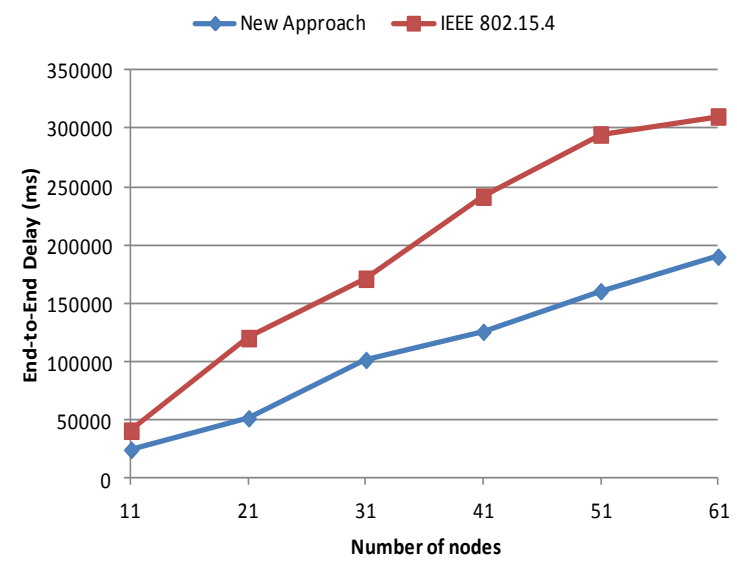

Figure 9: End-to-End delay for different network sizes

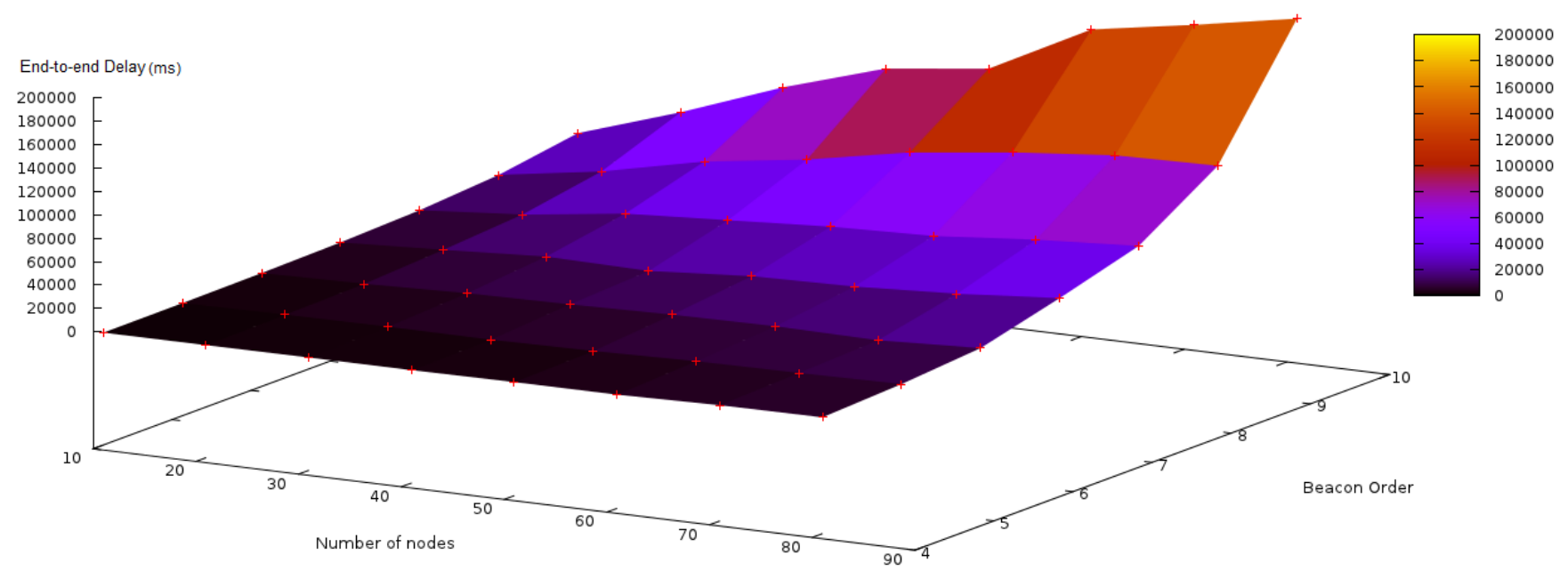

Figure 10: summary of all the scenarios for $\mathrm{SO}=4$ for the new proposed approach 


\section{CONCLUSION AND PERSPECTIVES}

In this paper we have described the IEEE 802.15.4 MAC protocol focusing on the beacon-enabled mode for the star topology. We showed that low duty cycle allows high power saving at the cost of high delay.

Previous research works on GTS of the IEEE802.15.4 have been focusing on increasing utilization and reducing the waste of bandwidth. However, we showed that the superframe of the current IEEE802.15.4 standard has some drawbacks especially for a very low duty cycle over a star topology. The enhanced structure of the superframe allows resolving the energy-delay trade-off in this kind of scenarios. The obtained simulation results using the NS-2 simulator show that the delay in our approach is decreased considerably compared to the GTS mechanism of the IEEE 802.15.4 standard for different levels of duty cycle and nodes numbers.

The presented results are encouraging and open many research perspectives. In the future works we plan to implement our approach on real-sensors using the iLive platform [20]. It is very important to test our proposal in real world environment. The protocol stack that will used is the Atmel open MAC [21] stack since it provides an open source implementation of the IEEE 802.15.4 standard. We also tend to improve and extend the proposed approach for multi-hop network, mainly the cluster tree topology.

\section{ACKNOWLEDGEMENT}

This project is supported by Franco-Moroccan cooperation program in information and communication science and technology research field and EU (FEDER)

\section{REFERENCES}

[1] I. Akyldiz, Y.S.W. Su, E. Cayirci, Wireless sensor networks: a survey, Comput. Netw. 38 (4) (2002) 393422. March.

[2] A. Mainwaring, J. Polastre, R. Szewczyk, D. Culler, "Wireless sensor networks for habitat monitoring," in: The ACM Workshop on Sensor Networks and Applications, 2002, pp. 88-97.

[3] IEEE Computer Society. 802.15.4 IEEE Standard for Information technology, 2006.

[4] W. Ye, J. Heidemann, D. Estrin, An energy-efficient MAC protocol for wireless sensor networks, IEEE Infocom. (2002) 1567-1576.

[5] T. van Dam K. Langendoen, "An adaptive energy efficient Mac protocol for wireless sensor networks," in: The First ACM Conference on Embedded Networked Sensor Systems (SenSys), pp. 171-180, November 2003.

[6] S.-H. Yang, H.-W. Tseng, E.-K. Wu, G.-H. Chen,"Utilization based duty cycle tuning MAC protocol for wireless sensor networks", GLOBECOM 20056 (5) (2005) 3258-3262.

[7] T. H. Hsu and J. S. Wu, "An Application-Specific Duty Cycle Adjustment MAC Protocol for Energy Conserving over Wireless Sensor Networks," Computer Communications, Vol. 31, No. 17, November 2008, pp. 4081-4088.

[8] H. Yong-Geun, K. Hyoung-Jun, P. Hee-Dong, and K. Do-Hyeon, "Adaptive GTS allocation scheme to support
QOS and multiple devices in 802.15.4," in Proceedings of the 11th International Conference on Advanced Communication Technology (ICACT '09), vol. 3, pp. 1697-1702, February 2009.

[9] Cheng, X. Zhang, and A. G. Bourgeois, "GTS allocation scheme revisited," Electronics Letters, vol. 43, no. 18, pp. 1005-1006, 2007. View at Publisher

[10] K. Anis, A. Mario, T. Eduardo, and C. Andre, "An implicit GTS allocation mechanism in IEEE 802.15.4 for time-sensitive wireless sensor networks: theory and practice," Real-Time Systems, vol. 39, no. 1-3, pp. 169204, 2008

[11] S. JunKeun, R. Jeong-Dong, K. SangCheol, K. JinWon, K. HaeYong, and M. PyeongSoo, "A dynamic GTS allocation algorithm in IEEE 802.15.4 for QoS guaranteed real-time applications," in Proceedings of the IEEE International Symposium on Consumer Electronics (ISCE '07), pp. 1-6, June 2007

[12] Z. Youling, W. Yi, M. Jianhua, J. Junpin, and W. Furong, "A low-latency GTS strategy in IEEE802.15.4 for industrial applications," in Proceedings of the 2nd International Conference on Future Generation Communication and Networking, (FGCN '08), vol. 1, pp. 411-414, December 2008

[13] Adrien van den Bossche, Thierry Val, Eric Campo, "Modelisation and validation of a full deterministic medium access method for IEEE 802.15.4 WPAN" Ad Hoc Networks 7 (2009) 1285-1301

[14] H. Kim, S.-G. Min, Priority-based QoS MAC protocol for wireless sensor networks, in: IPDPS '09: Proceedings of the 2009 IEEE International Symposium on Parallel\& Distributed Processing, IEEE Computer Society, Washington, DC, USA, 2009,

[15] T.H. Kim, S. Choi, Priority-based delay mitigation for eventmonitoring IEEE 802.15.4 LR-WPANs, IEEE Communications Letters 10 (3) (2006) 213-215.

[16] A. Koubaa, M. Alves, B. Nefzi, Y.Q. Song, Improving the IEEE 802.15.4 slotted CSMA/CA MAC for timecritical events in wireless sensor networks, in: 5th international workshop on Real-Time Networks (RTN'06), Dresden, Germany, 2006.

[17] M. Youn, Y.-Y. Oh, J. Lee, Y. Kim, IEEE 802.15.4 based QoS support slotted CSMA/CA MAC for wireless sensor networks, in: International Conference on Sensor Technologies and Applications, SensorComm 2007, 2007, pp. 113-117.

[18] J. Zheng and Myung J. Lee, "A comprehensive performance study of IEEE 802.15.4," Sensor Network Operations, IEEE Press, Wiley Interscience, Chapter 4, pp. 218-237, 2006.

[19] Network Simulator 2, http://www.isi.edu/nsnam/ns/

[20] Hong-Ling Shi, Kun Mean Hou, Hai-Ying Zhou, Xing Liu, "Energy Efficient and Fault Tolerant Multicore Wireless Sensor Network: E2MWSN", International Conference on Wireless Communications, Networking and Mobile Computing - WiCom, pp. 1-4, 2011

[21] IEEE 802.15.4 MAC stack software for Atmel devices, http://www.atmel.com/tools/ieee802_15_4mac.aspx/ 